\title{
DYNAMICS OF FRAGMENTATION REACTIONS FROM PEAK SHAPES IN MULTIPARTICLE COINCIDENCE EXPERIMENTS
}

\author{
J. H. D. ELAND \\ Physical Chemistry Laboratory, South Parks Road, Oxford, UK
}

\begin{abstract}
An experimental survey of peak shapes in two-parameter mass spectra from charge separation of doubly charged ions has been combined with Monte-Carlo simulations of peak shapes for different mechanisms. As a result, the major mechanisms, deferred charged separation, secondary dissociation and concerted explosion, can now be recognised. Finer details and a number of recurrent peculiar peak shapes remain unexplained.
\end{abstract}

KEY WORDS: Dynamics, coincidence, mass spectrometry.

\section{INTRODUCTION}

When a molecule breaks into just two fragments, the case usually discussed in molecular dynamics, the linear momenta given to the two fragments are precisely equal and opposite. Exact anticorrelation of the momenta is forced by the conservation law, and is of no dynamical interest. When a molecule breaks into three or more fragments, by contrast, any correlations between the particle motions reflect the mechanism of the reaction. Such correlations are uniquely manifested in the multiparameter peak shapes of charge separation mass spectrometery (CSMS). ${ }^{1}$ Some aspects of the peak shapes have been discussed in detail before, ${ }^{2}$ others are covered in a very recent thesis ${ }^{3}$ and more will be the subject of a forthcoming paper. ${ }^{4}$ Here we discuss the main peak shape types, and illustrate them from CSMS spectra of small molecules under photon and electron impact.

\section{KNOWN PEAK SHAPES}

The shape of a CSMS peak is a distribution of intensity as a function of the initial momentum components (along the spectrometer axis) of two, three or more ionic fragments from a charge separation. Initial momenta can be deduced from the peak shapes, and are those of the ions outside the zone of coulomb repulsion, that is, after the charge separation but before the applied electric field has had any significant influence. The most effective method of analysis is to simulate proposed mechanisms using Monte-Carlo techniques, and to fit the observed peak shapes. Because 


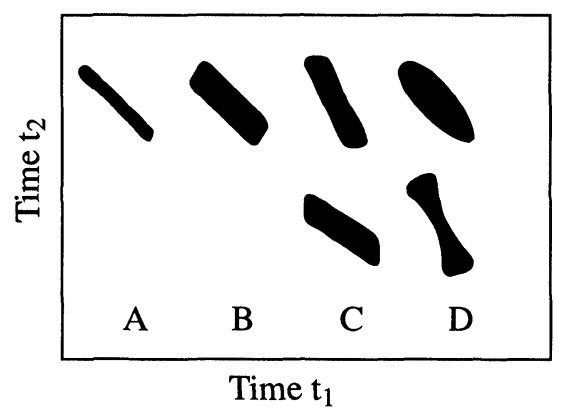

Figure 1 Simulated standard peak shapes for prototype mechanism: (a) two-body dissociation; (b) deferred charge separation; (c) secondary decay producing the lighter ion (upper) or heavier ion (lower shape); (d) concerted explosion with unseen fragment momentum perpendicular (upper shape) or parallel (lower) to the separation direction of the ions.

parameters such as energy releases or interparticle angles are always distributed rather than single-valued, this technique is essential.

From simulations and an ongoing survey in which CSMS spectra of about 50 compounds have been recorded, five basic peak types, illustrated in Figure 1, have emerged. The peaks are illustrated by their outer contours; the distribution of intensity within the peak is also important, but is governed mainly by the form of kinetic energy release distribution in each step of the reaction mechanism. In the following discussions the two observed ions are of masses $m_{1}$ and $m_{2}\left(m_{1}\right.$ lighter $)$ and $\mathrm{m}_{3}$ is the mass of an unobserved third fragment.

\section{Two-body Peaks}

The peak shape shown as " $A$ " in Figure 1 is a narrow bar of slope -1 with an intensity distribution perpendicular to its length derived from the thermal velocities of the molecules along the spectrometer axis before ionization. This shape is normally seen for parent ions breaking into two fragments, but may also appear in three-body reactions releasing a light neutral fragment with low momentum. The peak ends should have a definite slope (as in the next case), but this is seldom visible.

\section{Deferred Charge Separation}

Pairs of ionic fragments are often found to have momenta which are anticorrelated on average, but both ions also have a random momentum component, of the same sign and of magnitude proportional to mass for each ion of a pair. The peak shape, "B," is a lozenge of slope -1 and with ends of slope $m_{2} / m_{1}$ showing the correlation of the random components. The interpretation is that $m_{3}$ is ejected first leaving $m_{1} m_{2}^{2+}$, which rotates to a random angle relative to $m_{3}$ before breaking into two ions. Supporting evidence for this mechanism is often available in the form of an $m_{1} m_{2}^{2+}$ peak in the mass spectrum or a metastable tail in the spectrum demonstrating slow charge separation. ${ }^{5}$ Fine examples of this peak shape are seen in 


$$
\begin{aligned}
& \mathrm{SF}_{6}^{2+} \rightarrow \mathrm{SF}_{4}^{2+} \rightarrow \mathrm{SF}_{3}^{+}+\mathrm{F}^{+} \\
& \mathrm{CD}_{3} \mathrm{OD}^{2+} \rightarrow \mathrm{CD}_{2} \mathrm{O}^{2+} \rightarrow \mathrm{CDO}^{+}+\mathrm{D}^{+} \\
& \mathrm{NF}_{3}^{2+} \rightarrow \mathrm{NF}_{2}^{2+} \rightarrow \mathrm{NF}^{+}+\mathrm{F}^{+}
\end{aligned}
$$

In many cases the ends of the peaks are rounded because correlated "random" momentum components released in the first step are obscured by the spread of energy releases in the second step; the mechanism can still be identified on the basis of the supporting evidence.

\section{Secondary Decay of Primary Fragments}

Two fragments, one ion and one neutral, may be found moving away from the third (ionic) fragment with equal velocities and in the same direction on average. The peak shape is a bar at slope $-\left(m_{1}+m_{3}\right) / m_{1}$ or $-m_{2} /\left(m_{2}+m_{3}\right)$ and ideally with horizontal or vertical ends respectively. The simple interpretation is that one primary fragment of charge separation has decayed at a random orientation after escaping from the coulomb zone, and the width of the bar gives the energy release in the secondary decay. Clear examples involving outer atoms or groups as final ions are

$$
\begin{aligned}
& \mathrm{HCN}^{2+} \rightarrow \mathrm{HC}^{+}+\mathrm{N}^{+} \quad \mathrm{HC}^{+} \rightarrow \mathrm{H}^{+}+\mathrm{C} \\
& \mathrm{NO}_{2}^{2+} \rightarrow \mathrm{NO}^{+}+\mathrm{O}^{+} \quad \mathrm{NO}^{+} \rightarrow \mathrm{O}^{+}+\mathrm{N} \\
& \mathrm{CH}_{3} \mathrm{COCOCH}_{2}^{2+} \rightarrow \mathrm{CH}_{3} \mathrm{CO}^{+}+\mathrm{CH}_{3} \mathrm{CO}^{+} \quad \mathrm{CH}_{5} \mathrm{CO}^{+} \rightarrow \mathrm{CH}_{3}^{+}+\mathrm{CO}
\end{aligned}
$$

The best evidence for this mechanism is exact agreement between the observed slope and a mass ratio; supporting evidence is presence of the intermediate as a primary ion with the same initial momentum, and observation of the same secondary decay reaction in several molecules.

In the CSMS spectra of the linear molecules $\mathrm{CO}_{2}, \mathrm{OCS}, \mathrm{CS}_{2}, \mathrm{HCN}, \mathrm{BrCN}$ and ICN, peaks of this form are found for ion pairs including the central atom, such as $\mathrm{C}^{+}$ $+\mathrm{S}^{+}$from $\mathrm{CS}_{2}$ and OCS, $\mathrm{C}^{+}+\mathrm{O}^{+}$from $\mathrm{CO}_{2}$ and $\mathrm{OCS}, \mathrm{C}^{+}+\mathrm{I}^{+}$from $\mathrm{ICN}$ and so on. In some of these cases the best interpretation may be an obstructed explosion, where the neutral outer atom simply impedes, but is not bonded to the central ion. In $\mathrm{CS}_{2}$ decay, for example, a hypothetical $\mathrm{CS}^{+}$intermediate ought to decay to $\mathrm{S}^{+}+\mathrm{C}$ as well as to the observed $\mathrm{C}^{+}+\mathrm{S}$, but this is not observed. The pair $\mathrm{S}^{+}+\mathrm{S}^{+}$is observed, but with a slope of -1 , showing its origin in the next mechanism.

\section{Fast Concerted Dissociation}

Dissociation may proceed so fast, and in the limit instantaneously, that any intermediate has no time to rotate before dissociating. The three fragment momenta must then be angularly correlated, and complex peak shapes can result. If the unseen fragment is ejected at a significant angle away from the line connecting the two observed ions, the peak shape will be ovoid, as shown at "D" (upper) in Figure 1. Allowable combinations of vector magnitude and orientation can produce peaks 
with almost any slope, and any ratio of minor to major axis. ${ }^{3}$ Peaks of this form are found very often where all three fragments are charged but only two ions are detected; examples are shown in Figure 2 from the electron impact multiple ionization of OCS. It is less common for the unseen fragment momentum from dissociative double ionization to be large enough to give a pronounced ovoid form, but examples are

$$
\begin{aligned}
& \mathrm{SO}_{2}^{2+} \rightarrow \mathrm{O}^{+}+\mathrm{S}^{+}+\mathrm{O} \\
& \mathrm{CH}_{2} \mathrm{I}_{2}^{2+} \rightarrow \mathrm{I}^{+}+\mathrm{CH}_{2}+\mathrm{I}^{+}
\end{aligned}
$$

If all three fragments moved colinearly after dissociation but the unseen partner carried a significant distribution of momentum, a fan-shaped peak of arbitrary slope, simulated as "D" (lower) in Figure 1 would be seen. No peaks unambiguously of this form have been found yet, though apparatus discrimination against ions of high sideways velocity may produce peaks of similar appearance. A single-valued colinear momentum of the neutral would give a narrow bar of non-standard but calculable ${ }^{2}$ slope: this seems unlikely, and no definite case has been identified. A concerted dissociation where a neutral fragment receives a momentum distributed around zero gives a bar-shaped peak of slope -1 , exactly as in deferred charge separation. The mechanisms can often be distinguished by other evidence, however, particularly on the behaviour of hypothetical intermediates. For $\mathrm{S}^{+}+\mathrm{S}^{+}$from $\mathrm{CS}_{2}$, for instance, no $\mathrm{S}_{2}^{2+}$ is detected, and the mechanism is probably concerted.

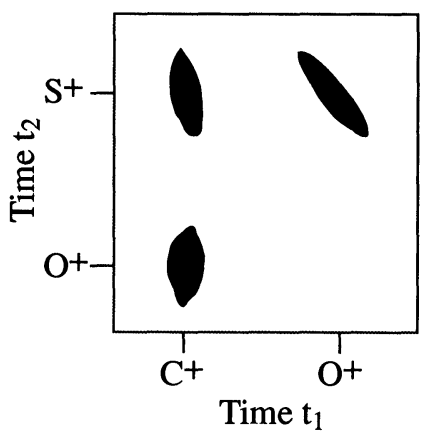

Figure 2 Peak shapes for the three possible ion pairs taken from experimental data on pure $\mathrm{C}^{+}+\mathrm{O}^{+}+$ $\mathrm{S}^{+}$triples formed by electron impact on OCS, showing ovoid form. The directly recorded pair spectra also show these shapes as aureoles around the real pair peaks from double ionization.

\section{MYSTERIOUS PEAK SHAPES}

The most interesting peak shapes are those we cannot yet explain. In spectra of both linear and non-linear molecules a few peaks of well-defined slope not equal to -1 or to a mass ratio have been found. An example is

$$
\mathrm{ICN}^{2+} \rightarrow \mathrm{I}^{+}+\mathrm{C}+\mathrm{N}^{+} \text {, slope }-1.23 \text { (Theor. }-1.86 \text { ) }
$$


Other mysterious peaks, mostly from the spectra of polyatomic molecules, have a twisted shape, in which the intense part of the peak has a different slope from the less intense part. Notable examples are

$$
\begin{aligned}
& \mathrm{N}_{2} \mathrm{O}^{2+} \rightarrow \mathrm{N}^{+}+\mathrm{O}^{+}+\mathrm{N} \\
& \mathrm{CF}_{2} \mathrm{ClBr}^{2+} \rightarrow \mathrm{CF}_{2}^{+}+\mathrm{Br}^{+}+\mathrm{Cl}
\end{aligned}
$$

The shapes show that a quantity determining peak slope is distributed rather than single-valued; possibilities are an energy release, an angle or a time. Lifetime distributions in secondary decay on a femtosecond timescale ${ }^{1,6}$ can fit the data, but the problem is to find the true explanation where many models are possible.

\section{References}

1. J. H. D. Eland, Acc. Chem. Res. 22, 381 (1989).

2. J. H. D. Eland, Mol. Phys. 61, 725 (1987).

3. T. LeBrun, Doctoral Thesis, Université Paris XI, 1991.

4. J. H. D. Eland and B. J. Treves-Brown, to be published.

5. J. H. D. Eland, F. S. Wort and R. N. Royds, J. Electron Spectrosc. Rel. Phenomena. 41, 297 (1987).

6. J. H. D. Eland and V. J. Murphy, Rapid Comm. Mass Spectrom. 5, 221 (1991). 